\title{
Prospective study to analyze the correlation between spontaneous bacterial peritonitis and model of end stage liver disease score
}

\begin{abstract}
Aim: To determine whether a greater MELD score is associated with a greater risk of development of spontaneous bacterial peritonitis.

Methods: This prospective study enrolled 148 consecutive patients with cirrhosis and ascites admitted between May 2008 to March 2010.After excluding patients who were Immuno suppressed, had history of prior antibiotic use, had previous episodes of SBP and had other confounding etiological factors for ascites, sixty seven patients were included in the study. SBP was defined as ascetic fluids PMN count $>250 / \mathrm{cu}$. mm. The odds ratio for development of SBP associated with MELD score and grouped MELD score was calculated $(<15,16-24,>25)$.
\end{abstract}

Results: The prevalence of SBP was $20.9 \%$.The mean MELD score in SBP group was23.14+/-9.87 and in the non SBP group was $18.46+/-7.43$. The odds ratio for development of SBP was 1.23 for each point rise in MELD score. $(p=.0032)$. Patients with MELD $>25$ had an odds ratio of $9.52(\mathrm{p}=0.001)$ for SBP as compared to patients with MELD $<15$

Conclusions: Increasing MELD score is independently associated with a greater risk of SBP. For every point increase in MELD score, the risk of developing SBP increases by $12.3 \%$. Prophylactic antibiotics should be considered in patients with MELD score $>25$.

Keywords: blood culture bottles, hiv infection, diabetes, cirrhosis, comorbid illness, high risk behavior
Volume 3 Issue 3 - 2017

Deepak Kumar Gupta

KEM Hospital, India

Correspondence: Deepak Kumar Gupta, KEM Hospital, West Minster building Room no 103 sector I6A, plot no 62, Nerul Navi Mumbai, India,Tel 932343 I40I, Email dkgt@rediffmail.com

Received: October 18, 2016 | Published: May 15, 2017

\section{Introduction}

Liver cirrhosis is a major cause of death all over the world. The major complications of cirrhosis include ascites, hepatic encephalopathy and hemorrhage caused by ruptured esophageal varices. Patients with cirrhosis and ascites have higher susceptibility to bacterial infections, mainly because of inadequate defence mechanisms. The most frequent infectious complications include Spontaneous bacterial peritonitis, Urinary tract infections, Pneumonia and Bacteremia. Since 1970, when SBP was first described and up to the present, the mortality rate has been decreasing from $80 \%$ to $30 \%$ due to prompt diagnosis and early initiation of adequate treatment. ${ }^{1}$

SBP is the infection of the ascitic fluid that occurs in absence of visceral perforation and in the absence of an intra-abdominal inflammatory focus. For SBP diagnosis, the polymorphonuclear leucocyte count in ascitic fluid should exceed $250 / \mathrm{mm}^{3}$ and from bacteriological cultures, only one organism should be isolated. ${ }^{1-4}$

\section{Methods}

The study was done at Jagjivanram Hospital, Mumbai in a prospective manner. All patients admitted with cirrhosis of liver and ascites from May 2008 to March 2010 were evaluated. Out of a total of 148 patients, eighty one were excluded. Reasons for exclusion were:
i. HIV infection (5 patients).
ii. Long standing diabetes/immuno suppressants (10 patients).

iii. Antibiotic use in two weeks prior to admission (53 patients).

iv. Confounding etiology for ascites (10 patients).

v. Previous history of SBP (3 patients).

Repeat admissions were not included. Patients with HIV infection, diabetes, immuno suppressants like steroids and alkylating agents may have been more predisposed to develop SBP regardless of their MELD score and so were excluded. Use of antibiotics in two weeks prior to admission may have prevented development of SBP or may have rendered paracentesis in sensivitive to the diagnosis of SBP .All patients with documented SBP are put on antibiotic prophylaxis in our centre and were excluded in the study. Sixty seven patients formed the study group.

\section{Evaluation of study groups}

a. Detailed history regarding symptoms, factors leading of cirrhosis, comorbid illness, high risk behaviour.

b. Lab investigations.

i. Complete hemorgram.

ii. PT/INR.

iii. Complete liver function tests, renal parameters, serum electrolytes.

iv. HIV $1 \& 2$, HBs Ag, anti HCV. 
v. USG abdomen.

vi. Ascitic fluid analysis and culture.

vii. Calculation of MELD.

SBP was defined as a paracentesis yielding $\geq 250$ neutrophils/ $\mathrm{ml}$ of ascitic fluid. Though bed side inoculation of fluid was done in blood culture bottles, a positive culture was not considered mandatory to diagnose SBP. The rationale was to capture both culture positive and culture negative neutrocytic ascites as both have similar natural history.

Patients with ascitic fluid PMN $<250$ cells $/ \mathrm{ml}$ were considered not to have SBP and found the control group. Data on subsequent paracentesis in same patients was not collected.

\section{Results}

Out of 67 patients who formed the study group, fourteen had SBP. The prevalence of SBP was $20.97 \%$.

Demographic Characteristics

\begin{tabular}{lll}
\hline Variable & Cases & Controls \\
\hline $\begin{array}{l}\text { No. of cases }(\mathrm{n}=67) \\
\text { \# age(yrs) }\end{array}$ & 14 & 53 \\
Mean & & \\
SD & 53.21 & 49.34 \\
Range & 10.58 & 9.95 \\
@Sex(\%) & $34-78$ & $23-78$ \\
Male & & \\
Female & $\mathrm{II}(78.6)$ & $43(81.1)$ \\
\end{tabular}

\# by Student ' $\mathrm{t}$ ' Test

(a)Chi-Square Test

$\mathrm{P}>0.05$ Not significant

In this study group age of the cases were ranging from 23-78years with mean age of 53.21years among cases and 49.34years in controls. $78.6-81.1 \%$ of the total cases were male in both the groups.

Profile of etiology of liver disease between the groups

\begin{tabular}{|c|c|c|c|c|c|}
\hline \multirow{2}{*}{ Etiology } & \multicolumn{2}{|c|}{ Cases $(n=14)$} & \multicolumn{2}{|c|}{ Controls $(n=53)$} & \multirow[t]{2}{*}{$P$ value } \\
\hline & No. & $\%$ & No. & $\%$ & \\
\hline Alcohol (42) & 10 & 71.5 & 32 & 60.4 & 0.447 \\
\hline Crypto (07) & 2 & 14.3 & 5 & 9.4 & 0.5976 \\
\hline $\mathrm{HCV}(07)$ & 1 & 7.1 & 6 & 11.3 & 0.6494 \\
\hline AlH (OI) & 1 & 7.1 & - & - & $* 0.0499$ \\
\hline $\mathrm{HBV}(06)$ & - & - & 6 & 11.3 & 0.187 \\
\hline $\mathrm{HBV}+\mathrm{HCV}(0 \mathrm{I})$ & - & - & I & 1.9 & 0.6045 \\
\hline$M D S+B C S(0 I)$ & - & - & 1 & 1.9 & 0.6045 \\
\hline NASH(02) & - & - & 2 & 3.8 & 0.4605 \\
\hline
\end{tabular}

By Chi - Square Test

$\mathrm{P}>0.05$ Not significant
Among cases, $71.5 \%$ had alcoholism as etiological cause for liver disease which was more than $60.4 \%$ in controls but difference was not significant. $11.3 \%$ controls had HBV as the reason for liver disease whereas none of the cases had this cause.

Comparison of changes in mean score of MELD between the groups

\begin{tabular}{|c|c|c|}
\hline \multirow{2}{*}{ Groups } & Mean score of MELD & \multirow{2}{*}{ Range } \\
\hline & $(\bar{x} \pm S D)$ & \\
\hline Cases & $23.14 \pm 9.87$ & Jun-36 \\
\hline Controls & $18.46 \pm 7.43$ & Jul-33 \\
\hline$P$ value & 0.1027 & \\
\hline
\end{tabular}

\section{By Chi - Square Test}

$\mathrm{P}>0.05$ Not significant

As per this table Mean MELD score in cases was 23.14 which were more as compared to 18.46 among controls but the difference was not significant statistically.

Profile of MELD grouping between the groups

\begin{tabular}{llllll}
\hline \multirow{2}{*}{ Groups } & \multicolumn{2}{c}{ Cases $(\mathbf{N}=14)$} & \multicolumn{2}{c}{ Controls( $\mathbf{N = 5 3 )}$} & \multirow{2}{*}{ P value } \\
\cline { 2 - 5 } & No. & $\%$ & No. & $\%$ & \\
\hline$\leq 15(n=24)$ & 3 & 21.4 & 201 & 38.5 & 0.2351 \\
$16-24(n=26)$ & 4 & 28.6 & 22 & 42.3 & 0.3505 \\
$\geq 25(n=17)$ & $* 07$ & 50 & 10 & 19.2 & 0.0194 \\
\hline
\end{tabular}

By Chi - Square Test

$\mathrm{P}>0.05$ Not significant

$50 \%$ of the patients in the SBP group had MELD $\geq 25$ compared to $19.2 \%$ in the SBP absent group (P 0.0194) Three patients with SBP had MELD score $\leq 15$.

$12.5 \%$ patients with MELD $\leq 15$ developed SBP. $41.17 \%$ patients MELD $\geq 25$ had SBP $(\mathrm{P}<0.001)$ and $15.38 \%$ patients with MELD between 16-24 had SBP $(\mathrm{P}<0.05)$.

The odds ratio for developing SBP by each MELD point were 1.23, $\mathrm{P}=0.0032$. Patients with MELD $>25$ had an odds ratio of 9.52(3.0133.59, $\mathrm{P}=0.001) \mathrm{SBP}$ as compared to subjects with MELD $<15$.

Profile of outcome between the groups

\begin{tabular}{|c|c|c|c|c|c|}
\hline \multirow{2}{*}{ Groups } & \multicolumn{2}{|c|}{ Cases(N=|4) } & \multicolumn{2}{|c|}{ Controls( $N=53)$} & \multirow{2}{*}{$\begin{array}{l}\mathbf{P} \\
\text { value }\end{array}$} \\
\hline & No. & $\%$ & No. & $\%$ & \\
\hline Survived $(n=57)$ & 10 & 71.4 & 47 & 88.7 & 0.1072 \\
\hline Expired $(n=10)$ & 4 & 28.6 & 6 & 11.3 & \\
\hline
\end{tabular}

By Chi - Square Test

$\mathrm{P}>0.05$ Not significant

This study report suggests that $88.7 \%$ SBP absent cases survived which were more than $71.4 \%$ but difference was not significant.

The mortality rate in SBP groups was nearly $30 \%$ compared to $11.3 \%$ in the SBP absent cases. The causes of death in SBP group were: Sepsis $^{2}$ and hepatorenal syndrome. ${ }^{2}$ In the control group, 
the causes of death were: UGI Bleed, ${ }^{3}$ Hepatorenal syndrome ${ }^{1}$ and Hepatic encephalopathy and aspiration. ${ }^{2}$

\section{Discussion}

Spontaneous ascitic fluid infection is a good marker of end stage liver disease and has been proposed as an indication for transplantation in a patient who is otherwise a candidate. In the 1980s the prevalence of SBP was approximately $27 \%$ but has dropped significantly now due to measures taken to prevent SBP. With better and faster treatment modalities, the mortality rate has also reduced significantly. ${ }^{5}$

In the study, greater MELD score was independently associated with a greater risk of SBP. Among patients admitted with cirrhosis and ascites, with every point increase in MELD the risk of developing SBP increased by $12.3 \%$. For those with MELD $\geq 25$, the risk of SBP was nearly ten times higher compared to those with MELD score $\leq 15$. This suggests that MELD score, a widely scoring system, is a useful tool to predict the presence of SBP.

In our group, three patients with MELD $\leq 15$ also had SBP $(21.4 \%)$. Thus, one cannot exclude the possibility of SBP solely on the basis of MELD score. This also substantiates the point that all patients with cirrhosis and ascites should undergo paracentesis at the time of admission to exclude SBP. The cell count should be reviewed as early as possible and antibiotics should be started if needed. Dipstick test results are available within 90-120seconds and may speed up treatment of SBP and improve survival. ${ }^{6}$

The study excluded patients who were at a higher risk of developing SBP like HIV infection, long standing diabetes and immuno suppressed patients with prior history of SBP and on antibiotics within 2weeks of admission. This helped us to correctly identify the relation between MELD score and development of SBP.

In the SBP group, mean MELD was 23.14 compared to MELD of 18.46 in the control group. This showed a trend towards significance. This shows that patients with advanced liver disease have higher chances of development of life threatening complications like SBP. Also, SBP per se can worsen hepatic and renal function and lead to higher MELD score.

Ascitic fluid was sent for culture in all patients. Three patients in SBP group had positive culture showing growth of E. coli. Thus, culture positivity was $21 \%$. Various studies have reported multiple organisms as the cause of SBP-E. coli (37\%), Klesiella pneumoniae(17\%), Pneumococci(12\%), Streptococcus viridans(10\%) and anaerobic organisms $(6 \%)$. Since culture positive and culture negative neutrocytic ascites have similar natural history, it is unlikely this would have affected the results. ${ }^{7-9}$

In a similar retrospective study done at the University of Pennysylvania, 111 patients were included in the study. Twenty nine of 111 hospitalised patients with cirrhosis were found to have SBP. The mean MELD score for patients with SBP was 24 and for those without $18(\mathrm{p}=0.0003)$. The odds ratio for developing SBP by each MELD point was $1.11(p=.001)$. Patients with MELD $\geq 25$ has an odds ratio of 9.67 for SBP compared to subject with MELD $\leq 15$. None of the potential confounders substantially altered the relationship between MELD and SBP. ${ }^{10}$

\section{Conclusion}

Diagnostic paracentesis should be done in all patients with cirrhosis and ascites to diagnoses SBP. Though patients with higher MELD score have a greater chance of developing SBP, one fifth of the patients with a MELD score of $<15$ developed SBP. Thus, paracentesis should be done at the earliest, preferably within one hour of admission to quickly diagnose and treat SBP.

Higher MELD score at presentation is associated with increased risk of development of SBP. Fifty percent of patients with MELD score more than 25 had SBP. The use of prophylactic antibiotics in these patients might be useful. At present, antibiotic prophylaxis is given to patients with previous documented episode of SBP or ascetic fluid albumin $<1 \mathrm{gm} / \mathrm{dl}$. Patients with a higher MELD Score have advanced liver disease and a higher risk of complications like infection, renal failure and encephalopathy. Thus, for these patients prophylactic antibiotics may be considered even if they have not developed SBP earlier.

Our study, with its limitations, makes a point about the need of antibiotic prophylaxis in high risk group of patients with MELD score $>25$. Larger studies and randomized controlled trials are required to make this a recommendation and prevent development of life threatening complications in this group.

\section{Acknowledgements}

None.

\section{Conflict of interest}

Author declares that there is no conflict of interest.

\section{References}

1. Guadalupe Garcia Tsao. Bacterial infections in cirrhosis : Treatment and prophylaxis. Journal of Hepatology. 2005;42(1):S85-S92.

2. Berg RD. Mechanisms promoting bacterial translocation from gastrointestinal tract. Adv Exp Med Biol. 1999;473:11-30.

3. Guarner C, Soriano G. Bacterial translocation and its consequences in patient with cirrhosis. Eur J Gastroenterol Hepatol. 2005;17(1):27-31.

4. Moore K. Spontaneous Bacterial peritonitis. In Warrel DA, editor. Oxford textbook of Medicine. 4th ed. India: Oxford University Press; 2003. p. 739-741.

5. Sort P, Navasa M, Arroyo V, et al. Effect of intravenous albumin on renal impairment and mortality in patients with cirrhosis and SBP. $N$ Engl $J$ Med. 1999;341(6):403-409.

6. Runyon BA. Strips and tubes: improving the diagnosis of spontaneous bacterial peritonitis. Hepatology. 2003;37(4):745-747.

7. Runyon BA. Monomicrobial nonneutrocytic bacterascites: a variant of spontaneous bacterial peritonitis. Hepatology. 1990;12(4 Pt 1):710-715.

8. Runyon BA, Hoefs JC. Culture negative neutrocytic ascites:a variant of spontaneous bacterial peritonitis. Hepatology. 1984;4(6):1209-1211.

9. Llovlet J, Rodriquez Iglesias P, Moitinho E, et al. Spontaneous bacterial peritonitis in patients with cirrhosis undergoing selective intestinal decontamination. J Hepatol. 1997;26(1):88-95.

10. Obstein KL, Campbell MS, Reddy KR, et al. Association between model of End stage liver disease and spontaneous bacterial peritonitis. Am J Gastroenterol. 2007;102(12):2732-2736. 